\title{
Young Mozart and the Joking Woody Allen Proper Names, Individuals and Parts
}

\author{
Matthias Paul \\ University of Edinburgh
}

\section{Introduction}

In semantic theory it is generally assumed that with simple sentences like the following ones we attribute properties or relations to some individual objects:

(1) The Rhine is polluted.

(2) W.A. Mozart visited Paris.

(3) Woody Allen is funny.

Herein the verbs specify the attributed properties or relations and the proper names the individual objects of which those properties or relations are claimed to hold. But consider now the following sentences which arise from (1) - (3) simply by adding some modifiers, here adjectives, to the proper names:

(4) The upper Rhine is polluted.

(5) The young W.A. Mozart visited Paris.

(6) The joking Woody Allen is funny.

It is obvious that (4) - (6) have different semantic contents from the corresponding ones in (1) - (3). For the sentences in the first group can all be true while the corresponding sentences in the second one are false. For instance with regard to (2) and (5) this could be the case if W.A. Mozart had visited Paris only in his later years. This allows us also to say the following without any contradiction:

(7) The young W.A. Mozart did not visit Paris but the old W.A. Mozart did.

Yet this sentence would be contradictory if we substituted W.A. Mozart for the two modified NPs or modified proper names, as we will call them. Thus the semantic contribution of such modified proper names must be different from the ones of the corresponding bare proper names. But what do those modified proper names contribute to the semantic contents of the sentences? And what are the contents of those sentences? Do we still attribute properties or relations to certain individuals?

In this paper it will be argued (i) that with sentences like (4) - (7) in which modified proper names occur we are not attributing anything to individual objects but to some of their parts and (ii) that the modified proper names denote those parts. Initial evidence for such a part-analysis comes from examples like (4) or (8):

(8) Eastern Scotland gets a lot of sun. 
which can be rephrased explicitly by using the noun part as in (9) and (10):

(9) The upper part of the Rhine is polluted.

(10) The eastern part of Scotland gets a lot of sun.

But on semantic grounds it will be argued that a part-analysis also works best for examples like (5) - (7) or the following one where a qualifying phrase is used with a proper name:

(11) The Paris of the 20ies was exciting.

In such cases we are not attributing a property to a spatial part of an individual as in (4) or (8) - (10) but to a certain temporal part.

In the first sections of the paper two alternative analyses of the above examples will be discussed: first, the adverbial analysis arising from the philosophical literature, for instance from Lowe (88), and second, Landman's (89) account which stems from his treatment of appositive as-phrases. Yet it will be argued that those analyses fall short on semantic grounds, i.e., they are neither compositional or uniform, nor are they able to explain the logical behaviour envinced by the above examples. As an alternative, in the following section the part-analysis will be worked out in detail. Under this analysis proper names denote sets of spatio-temporal parts of individuals as suggested by Quine (60). This will give us a semantically satisfying analysis of the above modified proper names under which they pick out certain parts of those sets. In the final section some prima facie objections against the part-analysis will be dealt with. In particular it will be shown that this analysis can be embedded into recent algebraic approaches suggested by Link (83) or Ojeda (91) that provide a unified semantics of common nouns.

\section{The Adverbial Analysis}

The surface structure of sentences (4) - (8) or (11) suggests that modifiers like young or qualifiers like in the 20ies are constituents of the resulting NPs, thus they should somehow affect their semantic values. Yet with regard to similar examples it has been argued that they have an adverbial status in the sense of specifying when and where some relation or property holds of some individual objects; see for instance Lowe (88) and also Haslanger (88) for such views. The modifiers are assumed to play the same semantic role as the prepositional phrases in the following examples:

(12) The Rhine is polluted in France.

(13) W.A. Mozart visited Paris in 1762.

Note that under this kind of approach we would with (4) - (6) attribute the same predicates or relations to the same individuals as we do in (1) - (3). By adding modifiers like young or upper we are simply specifying more in detail when Mozart visited Paris or where the Rhine is polluted. Evidence for this adverbial 
analysis seems to derive from the fact that for instance (5) can be rephrased as follows:

(14) W.A. Mozart visited Paris in his youth.

But despite this prima facie evidence I believe that the adverbial analysis fails on semantic grounds. First, it does not offer a compositional analysis nor a uniform one that fits in with other accounts of similar constructions. For example, one would expect that the determiner and the modifiers in (4) - (6) play the same role as they do in examples like the following ones:

(15) The young man is tall.

(16) The upper flat is the most expensive one.

But the role of the determiner simply gets ignored under the adverbial analysis and the noun modifiers are supposed to play the role of prepositional phrases or give rise to them in some mysterious way. Secondly, and even more importantly, the adverbial analysis seems unable to account for certain examples in which modified proper names occur. Consider for instance the following sentences with such NPs in object position:

(17) Mary likes the Paris of the 20ies

(18) John finds the young Bogart attractive.

With regard to such examples it seems implausible to say that the temporal modifiers or qualifiers specify the time when some property or relation holds of a certain individual. For instance in order for (17) and (18) to be true neither must Mary have liked Paris in the 20ies nor must John have found Bogart attractive in Bogart's youth. In fact the truth of (17) and (18) is compatible with the truth of the following sentences:

(19) Mary started to like the Paris of the 20ies after visiting the 1993 Paris exhibition.

(20) John only recently started finding the young Bogart attractive .

To summarize, the account of modified proper names given by the adverbial analysis neither seems to be uniform nor compositional, and even more importantly it yields the wrong contents for examples like (17) and (18), i.e., it makes wrong predictions regarding the logical behaviour of certain examples. ${ }^{1}$

\section{Restricted NPs and Intensional Individuals}

In his paper "Groups II", Landman deals with intensional plural expressions like committee. He proposes to treat them analogously to the NPs in the following sentences which consist of a proper name and a qualifying as-phrase:

(21) John as a judge is trustworthy. 
(22) John as the president of the football club is corrupt.

Note that although John as a judge can be trustworthy he might not be so as the president of the football club, i.e., the truth of (21) is compatible with that of (22). Or to put it differently: substitution of the NPs in (21) and (22) for each other is not truth preserving, thus their semantic contribution seems to be different and in particular different from the one of the bare proper name John. It is obvious that examples (21) and (22) strongly resemble the ones we discussed in previous sections. For adding as-phrases to proper names and turning them into complex NPs has the same semantic effect as it had in the above examples where we added other kinds of modifiers or qualifiers.

Landman proposes to treat examples (21) and (22) as intensional contexts in which we do not attribute a property to an individual as such, but only under a certain aspect, i.e., to an intensional individual as he calls it. Then sentences (21) and (22) can both be true because the NPs in them denote different intensional individuals. What gives rise to the intensionality of these examples are the appositive as-phrases which are assumed to restrict the denotation of the NPs in a particular way. According to Landman such restrictions can also be made implicitly as for instance in the following examples:

(23) The judge is on strike.

(24) The judge is the hangman.

(25) The hangman is on strike.

Sentences (23) and (25) constitute intensional contexts since it is possible that (23) and (24) can both be true while (25) is false. The only difference to the above example is that the NPs in (23) and (25) involve some hidden restrictions that give rise to this intensionality which for instance with regard to (23) can be made explicit as follows:

(26) The judge as a judge is on strike.

Landman formalizes intensional individuals as set of properties. For an extensional individual like John the usual correspondence between the individual itself and the set of all its properties holds, i.e., predicating something of John can be restated in terms of predicating something of the following set of properties:

(27) $\{\mathrm{PI}$ P(john) $\}$.

However for restricted NPs like John as a judge which are assumed to give rise to intensional contexts we do not have such a correspondence. They are assumed to denote sets of properties which are different from ones like (27). Landman defines some plausible closure conditions on such sets which turn restricted NPs into ultrafilters of properties. This leads to an intensional treatment of properties in the model of the language since in any extensional framework the set of properties denoted by a restricted term like John as a judge would equal (27) and that is 
something we clearly do not want; see Landman (89) for a proof of this feature. As part of his intensional model Landman assumes a function of restriction, let us call it $f_{\text {res }}$, that takes an individual and a property and maps it onto a set of properties. Then, if we have a restricted NP like John as a judge, its semantic value is given as follows:

(28) $[[$ John as a judge $]]=f_{\text {res }}$ (john, Judge).

Now, it seems that depending on the way this restriction function is defined we can get different denotations for the NPs in (21) and (22). But it is not clear which values $\mathrm{f}_{\text {res }}$ will give us. For as far as I can see we do not have a notion of an individual having a property as something or under a certain aspect. Rather we think of individuals as having properties simpliciter or relative to times as in John was tired this weekend. But let us assume for a moment that this notion could be illuminated further. Then the central question becomes whether a Landman-style analysis also seems suitable for examples (4) - (8) or (11). According to such an analysis, we would be dealing here with intensional contexts where the modified proper names like The upper Rhine or The young Mozart function as restricted NPs in the sense of denoting certain sets of properties. Clearly, given such kinds of denotations we would get enough "finegrainedness" to account for the logical behaviour of the constructions in which they occur. But the problem is that those examples do not seem to constitute intensional contexts. For instance it seems impossible that (4) and (29) are true while (30) is true is well:

(4) The upper Rhine is polluted.

(29) The French Rhine is not polluted.

(30) The upper Rhine is (identical with) the French Rhine.

Whenever (4) and (29) are true (30) must be false. The same case can be made with regard to temporally modified proper names. For instance (7) and (31) cannot both be true:

(7) The young W.A. Mozart did not visit Paris yet the old W.A. Mozart did.

(31) The young W.A. Mozart is (identical with) the old W.A. Mozart.

Thus, in summary, a Landman-style analysis of the modified proper names in (4) (8) or (11) seems implausible on semantic grounds. Not only is it unclear which intensions such NPs would denote, but more importantly, those sentences do not seem to constitute intensional contexts; they fail standard intensionality tests. In the following I will show how the part-analysis can give an extensional analysis of these NPs that treats them on a par with structurally identical constructions.

\section{The Part-Analysis}

The part-analysis claims that sentences like (4) - (8) in which modified proper names occur can be best accounted for by assuming (i) that we are not making any 
statements about individual objects but about some of their parts and (ii) that the modified proper names denote those parts. This would gives us contents of the following kind for (4) - (6):

(32) Polluted(upper_part_rhine);

(33) Visited-Paris(young_part_w.a.mozart);

(34) Funny(joking_part_woody.allen).

where for instance upper_part_rhine stands for the upper part of the Rhine. It is clear that under such an analysis the logical behavior of constructions like (4) - (6) can be explained easily. For instance (5) and (35a) can both be true and not lead to any contradiction since we would make statements about different parts of Mozart as envinced by (35b):

(35) a. The old W.A. Mozart did not visit Paris.

b. $\neg$ Visited-Paris(old_part_w.a.mozart).

Further support for the analysis stems from the fact that parts of individuals can be denoted directly by NPs like The upper part of the Rhine, as noted already above.

Yet, as it stands, the case for the part-analysis does not seem that strong.

First, with regard to temporally modified NPs like The young W.A. Mozart, paraphrases involving the notion part explicitly are hard to come by. Secondly, as we have seen in the previous section, the logical behavior of (5) and (35a) can also be explained under a Landman-style analysis. Thus further arguments are needed for the part-analysis. One has to account for the notion of a part of an individual in order to illuminate the claim that The young W.A. Mozart can denote such an entity. It would also be desirable if a compositional and uniform account for such modified proper names could be given that treats them semantically in the same way as structurally similar NPs like The young man or The upper flat in (15) and (16).

Starting with the latter question the first thing to note is that the NPs in (4) (6) and in (15) and (16) all have the following structure:

(36) a. [the [Mod Noun $\left.]_{N^{\prime}}\right]_{N P}$
b. [the [Noun Quall $\left.N^{\prime}\right] N P$

where Noun is a common or a proper noun, Mod or Qual a modifier or a qualifier and $N^{\prime}$ and $N P$ stand for the different types of categories. It is generally assumed that common nouns denote some sets of entities and modifiers or qualifiers denote functions that map those sets onto others set of entities of the same type, i.e., for an $\mathrm{N}$-constituent we get the following denotation:

(37) $\left[\left[N^{\prime}\right]\right]=\left\{x \mid x \in[[M o d / Q u a l]([[N o u n]])\}^{2}\right.$.

In most cases modifiers or qualifiers will be affirmative, i.e., the following holds:

(38) [[Noun]] $\supseteq[[\mathrm{Mod} / \mathrm{Qual}]]$ ([[Noun $])$. 
This seems to be the case with adjectives like young or old but also with eastern or upper; see Kamp (75) for other examples of affirmative adjectives. Now, under Russell's and Sharvy's (80) influential analyses of definite descriptions, expressions of the form $(36 \mathrm{a}, \mathrm{b})$ function as incomplete symbols that lead to the following claims:

(39) a. Russell $\exists ! x\{x\}=\left\{x \mid x \in\left[\left[N^{\prime}\right]\right]\right.$;

b. Sharvy $\exists x \quad x=\max _{\leq}\left\{x \mid x \in\left[\left[N^{\prime}\right]\right\}\right.$.

In (39a) a definite description asserts the existence of a unique element in the set denoted by the N'-constituent whereas in (39b) it asserts the existence of a greatest or maximal element in this set that is according to Sharvy partially ordered by some part relation $\leq ; \mathrm{a} \in\left[\left[\mathrm{N}^{\prime}\right]\right]$ is the maximal element in [[N']] if for all $\mathrm{x} \in\left[\left[\mathrm{N}^{\prime}\right]\right] \mathrm{x} \leq \mathrm{a}$. The main advantage of Sharvy's analysis is that it allows for a unified treatment of the different kinds of common nouns, i.e., for singular and plural count nouns as well as for mass nouns. This is the reason why it will be adopted it in the following. ${ }^{3}$ But instead of treating definite description of the form (36a, b) as incomplete symbols we will treat them as referring expressions with the following Sharvy-style semantics:

(40) $\left[\left[\right.\right.$ the $\left.\left.N^{\prime}\right]\right]=\max \leq\left\{x \mid x \in\left[\left[N^{\prime}\right]\right\}\right.$ if it exists, otherwise undefined.

Now, if we could specify some sets as denotations for proper names that are ordered under a part-relation, this Sharvy-style semantics would be applicable, thus we would get a compositional and uniform treatment for modified proper names like The upper Rhine. But the problem is that not just any set will do as denotation for a proper name like The Rhine. Consider for instance the following set as its denotation:

(41) $[[$ Rhine $]]=\{x \mid x=$ rhine $\}$.

This is a singleton set containing the river Rhine, thus combined with an affirmative modifier like upper, the denotation of upper Rhine could according to (38) only be that set or the empty set. But this leads to the problem that we would not get as denotation for The upper Rhine any proper part of the Rhine. Thus some other kind of set is needed to provide denotations for proper names. What comes to mind here is the following: if we conceive of individuals as entities that have parts a proper name can denote the set of all those parts. Then the above Sharvy-style analysis will in principle yield the right results, i.e. certain parts of individuals as denotations for NPs like The young Mozart or The upper Rhine. The function of adjectives like young or upper would simply be to pick out the set of those parts of an individual that are young or upper parts and the definite article would give us the maximal element in those sets, for example for The upper Rhine the maximal upper part of the Rhine.

This leads us to the question whether we can motivate a conception of individuals under which they consist of parts and are not atomic entities as is 
usually assumed. The notion of an individual I want to appeal to here is that of a spatio-temporal region or "sausage" as outlined by Goodman (51) and Quine (60) and used for semantic purposes by Carlson (77) and Hinrichs (85). Regarding such entities it makes sense to say that they have parts, namely spatio-temporal parts, which can be seen as different slices of such sausages, as time slices or spatial slices. For instance W.A. Mozart's arm will be a particular spatial slice of the sausage W.A. Mozart whereas the young Mozart will be a certain temporal slice. Such parts can have other spatio-temporal parts as parts. For instance Southern Germany which is part of Germany has München and Stuttgart as parts, or the young W.A. Mozart which is part of W.A. Mozart may have the child Mozart and the teenager Mozart as parts. Also parts of an individual can be merged or fused together such that the resulting entity will again be a part of that individual. For instance different time slices of Paris, let us say its night slices, can be merged and the resulting part will again be a part of Paris. Finally ordinary properties and relations like Polluted or Funny as envinced by (32) and (34) can hold of such spatio-temporal parts, for instance Polluted of the upper Rhine or Funny of the joking Woody Allen. In fact the usual notion of a property $\mathrm{P}$ holding of an individual $i$ at a time $t$, i.e. $P(i, t)$, can be reduced to the following: there is a part $p$ of $i$ such that p's temporal extension is $t$ and $P$ holds of $p$, i.e. $P(p)$. This conception of individuals as complex objects can be made more precise as follows: 4

(42) a. D is a non-empty set, namely our semantic domain, and ID a non-empty subset of $D$, namely the set of all individuals $i, i$,' $i ", \ldots$ in $D$;

b. D is partially ordered by a spatio-temporal part relation $\leq_{\mathrm{st}}$, such that $\leq_{\text {st }}$ is reflexive, transitive and antisymmetrical;

c. For each $i \in I D$, there is a set $P_{i}:=\left\{x \mid x \leq_{s t} i\right\}$ of $i$ 's parts which contains more elements than just $i$ and forms a complete join semilattice under $\leq_{\text {st }}$, i.e., it is partially ordered by $\leq_{s t}$ and each non-empty subset $\mathrm{P}^{\prime}$ of $\mathrm{P}_{\mathrm{i}}$ has a supremum in $\mathrm{P}_{\mathrm{i}}$.

On the basis of this conception of individuals it makes sense to say that a proper name denotes a set of parts. For as Quine (60) suggested, it simply denotes the set $\mathrm{P}_{\mathrm{i}}$ of spatio-temporal parts of an individual $\mathrm{i}$, where the individual itself is the maximal element in such a set. Thus a proper name like Mozart functions more like a noun or general term in the sense of denoting all those entities that make up Mozart or that are "Mozartizers". Let us assume with Sharvy(80) that for each noun there is a part-relation $\leq$ given on its extension which provides a partial order on that set. For proper names this part relation is the spatio-temporal part relation $\leq_{\text {st }}$ as described above which partially orders the union of all extensions of proper names; for mass nouns there might be different part relations. Further assume that all noun-modifiers and -qualifiers are affirmative, thus the denotations of $\mathrm{N}^{\prime}$ constituents will also be partially ordered by the part relations of their head nouns. Then a model for our language can be defined as the structure $<D, I D,[I], f_{\leq}>$ where:

a. D and ID satisfy the conditions stated under (42); 
b. [[ ] is an interpretation function that maps nouns and intransitive verb phrases onto subsets of $\mathrm{D}$, in particular proper nouns onto some $\mathrm{P}_{\mathrm{i}}$ for an individual $\mathrm{i} \in \mathrm{ID}$, and modifiers and qualifiers onto functions from $\mathrm{D}$ to $\mathrm{D}$;

c. $\mathrm{f}_{\leq}$is a function that gives for each noun $\mathrm{N}$ a partial order on [N]; for proper nouns this partial order will be given by $\leq_{\mathrm{st}}$.

The first thing to note is that the model provides the right denotations for modified proper names in examples like (4) - (8) or (11) assuming we add (37) and (40) as semantic rules for N'-constituents and NPs. For instance the NP The Young W.A. Mozart will denote the maximal element in the set of entities that are Mozartizers and young under the spatio-temporal part relation $\leq_{\text {st }}$, i.e., we get the following:

(44) $[[$ The young W.A. Mozart $]]=\max _{\leq \mathrm{st}}\{\mathrm{x} \mid \mathrm{x} \in[[$ Young $]([[$ W.A. Mozart $]])\}$.

This denotation will be different from the one we get for The old W.A. Mozart since the adjectives will pick out different parts of the individual Mozart, young only young parts and old only old parts. For the NP The upper Rhine the analysis yields that part of the Rhine which is its maximal upper part and for The joking Woody Allen that part of Woody Allen which is his maximal joking part; the latter will be the fusion of all those temporal slices or stages of Woody Allen that are joking since the fusion is the maximum of all those joking Woody Allen parts.

Further note that the part-analysis not only provides the right denotations for modified proper names but also treats them analogously to similar NPs with common nouns in head position. For instance for the NP The young cheese we get the same kind of denotation, although the part relation might be a different one here:

(45) [[The young cheese $]=\max _{\leq}$cheese $\{\mathbf{x} \mid \mathbf{x} \in[$ Y Young $]$ ([ICheese $\left.\left.]\right)\right\}$.

In summary, the part-analysis yields the right kind of denotations for the modified proper names in (4) - (8) and (11) while not treating them in any way differently from other kinds of definite NPs. In particular it yields a compositional account, thus it succeeds on semantic grounds. ${ }^{5}$ In the following section some prima facie objections to the part- analysis will be discussed.

\section{Some Prima Facie Objections}

As it stands a Sharvy-style analysis of definite NPs does not apply to NPs like Eastern Scotland or Mozart where the definite article is missing; such NPs for instance occur in sentences (2) and (8). Following Burge (73) and Bach (87) I suggest that we should analyse them semantically by positing a null-determiner. In particular, assuming that the null-determiner is a definite article, the Sharvy-style analysis under (40) can be applied. It will yield the right kind of denotations, for instance for the NP W.A. Mozart the individual Mozart since the following holds (see also Sharvy (80) for this analysis of proper names as full NPs): 
(46) $[[[\text { W.A. Mozart }]]]_{\mathrm{NP}}=\max _{\leq \mathrm{s}} \mathrm{P}_{\mathrm{w} \cdot \mathrm{a} \cdot \mathrm{mozart}}=$ w.a. mozart.

Note that modified or unmodified mass nouns can also function as full NPs in themselves as the following examples show:

(47) Cheese tastes good.

(48) Young cheese tastes good.

With regard to such constructions that have no definite article, one also wants to specify the denotations by a Sharvy-style analysis of definite NPs. Thus there is nothing ad hoc if we do the same regarding the structurally similar NPs in (2) and (8). In some contexts proper names just seem to function syntactically more like mass nouns, in others more like count nouns. ${ }^{6}$

The final point I want to make relates to the recent algebraic approaches to providing a unified semantics of common nouns as suggested by Link (83) and worked out further by Ojeda (91). For one might think that the above analysis of proper names blurs an explanatorily important classification of the different kinds of common nouns as put forward by those authors. This classification is based on the assumptions that the semantic domain D in (43) forms a complete join semilattice under a part relation $\leq$; in the following we will write $+D^{\prime}$ for $\sup _{\leq} \mathrm{D}^{\prime}$ and call + the join over the elements in $\mathrm{D}^{\prime}$, with $\mathrm{D} \supseteq \mathrm{D}^{\prime} .^{7}$ Then one can define the notion of a minimal element and some other related notions relative to $\mathrm{D}$ as follows:

a. An element $\mathrm{a} \in \mathrm{A}, \mathrm{D} \supseteq \mathrm{A}$, is a minimal element or atom in $\mathrm{A}$ iff for all $\mathrm{b} \in \mathrm{A}$ if $\mathrm{b} \leq \mathrm{a}$ then $\mathrm{a}=\mathrm{b}$;

b. An element $a \in A, D \supseteq A$, is a molecule if it is the join of some nonempty set of atoms of $A$, i.e. $a=+A^{\prime}$ with $A^{\prime}$ is a set of atoms;

c. An element $\mathrm{a} \in \mathrm{A}, \mathrm{D} \supseteq \mathrm{A}$, that is neither a molecule or an atom is a nonmolecule.

Ojeda (89) proposes that singular and plural count nouns as well as mass nouns can be classified by the different kinds of entities they denote. All will denote some subset of our semantic universe D but the subsets will differ as follows:
a. A singular count noun $\left(\mathrm{CN}_{\text {sing }}\right)$ denotes a set of atoms of $\mathrm{D}$;
b. A plural count noun $\left(\mathrm{CN}_{\text {plur }}\right)$ denotes a set of molecules of $\mathrm{D}$;
c. A mass noun (MN) denotes a set of nonmolecules of $\mathrm{D}$.

Now the problem is that this classification rests on the assumption that individuals like W.A. Mozart or Woody Allen are atoms in D since they are supposed to be in the denotation of singular count nouns. But under the above part-analysis they are treated as complex entities consisting of parts. Hence the model stated in (43) undermines the classification of common nouns in (50). For if individuals are not atoms in D, singular count nouns which are supposed to denote sets of individuals will at least denote sets of molecules or possibly also sets of nonmolecules, 
depending on what kind of entities we conceive individuals to be. But fortunately there are ways of restoring order such as the following:

(51) A model for our language is a structure $<D$, ID, [ ] ] $>$ such that $<D, \leq,+>$ is a complete join semilattice, ID is subset of $\mathrm{D}$ and [[ ] ] an interpretation function as defined under (43) with the following further conditions:

(a) [[ $\left.\mathrm{CN}_{\text {sing }}\right]$ is a subset of ID;

(b) $\left[\left[\mathrm{CN}_{\text {plur }}\right]\right.$ is a subset of $\{\mathrm{x} \mid \mathrm{x}=+\mathrm{A}, \mathrm{ID} \supseteq \mathrm{A}\}$;

(c) [IMN]] is a subset of $\{x \mid x=+A, A \cap D \backslash\{y \mid y=+B, I D \supseteq B\} \neq \varnothing\}$;

(d) [[PN] is a subset $P_{i}:=\{x \mid x \leq i\}$ of $D$ with $i \in I D$.

The set ID is the set of individual objects as outlined above relative to which a similar classification of nouns can be made as under (50). If we take ID as our set of atoms then singular count nouns denote relative to ID sets of atoms, plural count nouns sets of molecules that have those atoms as parts and mass nouns sets of entities of which some are neither atoms nor molecules relative to ID. In addition we have the extra condition that proper nouns denote subsets of $\mathrm{D}$, namely sets of parts of individuals as stated under (42) and (43). Thus the above treatment of proper names does not blur the classification in (50) but rather enriches it in the sense that we get a general account of nouns.

\section{Conclusion}

In this paper I have proposed a part-analysis for sentences like (4) - (8) and (11) in which modified proper names occur. According to this analysis we are attributing herein properties or relations to particular spatio-temporal parts of individuals that are denoted by the modified proper names like The young Mozart or The upper Rhine. Central to the part-analysis is a conception of individuals as complex objects consisting of spatio-temporal parts. This allows for a Quinean treatment of proper names under which they denote sets of parts of an individual. Then the function of the modifiers becomes to pick out a subset of such a set and combined with a Sharvy-style analysis of definite descriptions we get as denotations the maximal element in such a set. From a semantic perspective the proposed analysis seem preferable to others since it not only enables us to explain the logical behavior of sentences in which modified proper names occur but also does so in a compositional and uniform way which treats The young Mozart on a par with structurally similar NPs like The young man or The young cheese. What emerges is a unified semantic analysis of common and proper nouns according to which they just denote different kinds of subsets of our semantic universe D.

\section{Endnotes}

* I want to thank Elisabet Engdahl, Jonathan Ginzburg and Robin Cooper; the first for encouraging my interest in the topic of the paper and the latter two for 
many helpful comments on different versions of it. The paper has also profited from the participants of SALT4 and of several workshops and discussion groups in Edinburgh, notably from comments by Ivan Derzhanski, Alice Drewery, Sheila Glasbey, Massimo Poesio, Lucia Tovena and Peter Ruhrberg.

1. Note that even if semantic considerations played no role in judging the adequacy of the adverbial analysis it is highly questionable if it can achieve its own underlying aim which seems to be the avoidance of parts of individual objects in the semantics of sentences like (4) - (8) or (11). For instance examples involving spatial modifiers like (4) or (8) cannot be rephrased in the same way as (5). Their most suitable paraphrases seem to be the following ones which both explicitly mention parts of individuals:

(a) The Rhine is polluted in its upper part.

(b) Scotland gets a lot of sun in its eastern part.

Thus we have at least to allow for spatial parts in our semantics. But even with temporal modifiers as in (5) or (6) things are not as straightforward as one might expect. For instance what do we denote with in his youth in (14)? We do not seem to refer to any external time instant or interval as we do in (13) with in 1762. Thus here a case could be made for temporal parts of individuals. But if we have to assume parts anyway in our ontology one might wonder why not to allow the modified proper names to denote them directly as suggested under the partanalysis.

2. The sets of entities denoted by nouns need not necessarily be subsets of our semantic domain D but might be subsets of the power set of D. This seems plausible for instance with regard to plural count nouns like dogs or cats; see Landman (89) for such an account of common nouns. But in order to simplify our presentation I will assume in the following that the sets denoted by the different kinds of common nouns are always subsets of D; see Link (83) or Ojeda (91) for accounts that make this assumption.

3. For mass nouns and plural count nouns Russell's analysis seems inadequate since the $\mathrm{N}$ '-constituents here do not have to denote singleton sets. For instance the $\mathrm{N}^{\prime}$-constituent in The Gold in Zürich will denote a set that contains more than one piece of gold since there will be many of them in Zürich. The function of the definite article here is not to indicate uniqueness but totality, i.e., to pick out the maximal element that is gold in Zürich (see Sharvy (80) and Ojeda (91) for more discussion of this issue). In recent years a Sharvy-style analysis of definite descriptions has been adopted in some algebraic approaches to the semantics of common nouns; see for instance Ojeda (91).

4. Carlson (77) makes use of the notion of a spatio-temporal part in order to account for the two readings one gets for bare plurals, i.e. the existential and the universal reading. For instance a bare plural like rain drops gives rise to the existential reading in Rain drops fell on the city and to the universal reading in Rain drops are wet. In the first case we are supposed to existentially quantify over spatio-temporal parts of individuals, or stages as he calls them, whereas in the second case we predicate something of complex individual objects. What gives rise 
to those readings are, according to Carlson, the different kinds of predicates that combine with a bare plural, either a stage-level predicate or an individual-level predicate. It should be noted that the part-readings we get for the NPs in sentences like (4) - (8) do not depend in any way on the involved predicates and this is one of the reasons why a Carlson-style explanation in terms of stage-level predicates is not available to us here.

5. The part-analysis might offer an alternative account of the Landman examples such as John as a judge. For instead of treating sentences in which such NPs occur as intensional contexts, which seems disputable anyway, one could give an extensional treatment under which the NPs just pick out certain parts of the sets denoted by the proper names. For instance John as a judge would denote the maximal temporal judge-acting part of John. Clearly, such a part would be different from his maximal football-club-president-acting part which would be denoted by John as the president of the football club.

6. Burge and others noted that proper names sometimes seem to function more like count nouns as suggested by the following examples The Alfred who plays the flute joined the band today or I know few Alfreds in Edinburgh. In the light of this evidence Burge proposed to treat proper names as denoting sets of individuals and not denoting particular individuals. One should note that on the basis of some minor modifications this proposal can be fitted in very easily to the above treatment of proper names. The most reasonable option here is to relativize the interpretation function [[] to a context factor $\mathrm{c}$, i.e., we get $\left[\Pi_{\mathrm{c}}\right.$. Then relative to some $\mathrm{c}$ a proper name could denote a set of individuals but relative to another $c^{\prime}$ a set of parts of an individual. This would give us the right denotations for the resulting NPs in which those proper names occur as constituents.

7. Note that if one takes into account how is part of gets used in natural language it seems more plausible that there are different part relations as suggested under (43). But one can make sense of a unique part relation by conceiving of it more along the lines of a spatio-temporal or material part relation as suggested by Goodman (51) or Quine(60).

\section{References}

Bach, K. (1987) Thought and Reference, Clarendon Press Oxford.

Bunt, H. (1985) Mass Terms and Model-Theoretic Semantics, Cambridge University Press, Cambridge.

Burge, T. (1973) "Reference and Proper Names", Journal of Philosophy 70, 423439.

Carlson, G. (1977) "A Unified Analysis of the English Bare Plural", Linguistics and Philosophy 1, 413-456.

Goodman, N. (1951) The Structure of Appearance, New Edition (77), Dordrecht.

Haslanger, S. (1988) "Endurance and Temporary Intrinsics", Analysis 48, 119-25.

Hinrichs, E.W. (1985) A Compositional Semantics for Aktionsarten and NP Reference in English, PhD Dissertation, Ohio State University. 
Kamp, J.A.W. (1975) "Two theories about adjectives", in Keenan (ed.), Formal Semantics of Natural Language, Cambridge University Press, Cambridge. Landman, F. (1989) "Groups I, II", Linguistics and Philosophy 12, 559-605 \& 723-744.

Lewis, D. (1986) On the Plurality of Worlds, Cambridge Blackwell, Cambridge. Link, G. (1983) "The Logical Analysis of Plurals and Mass Terms: a LatticeTheoretic Approach", in Bäuerle, Schwarze, and von Stechow (eds.), Meaning, Use, and the Interpretation of Language, de Gruyter, Berlin.

Lowe, E.J. (1988) "The Problems of Intrinsic Change", Analysis 48, p. 72-77.

Ojeda, A.E. (1991) "Definite Descriptions and Definite Generics", Linguistics and Philosophy 14, 367-397.

Quine, W.V.O. (1960) Word and Object, MIT Press, Cambridge.

Sharvy, R. (1980) "A more General Theory of Definite Description", Philosophical Review 89, 607-624. 